\title{
AJUSTE DA FUNÇÃO DE DISTRIBUIÇÃO DIAMÉTRICA WEIBULL POR PLANILHA ELETRÔNICA
}

\author{
William Thomaz Wendling ${ }^{1}$, Dartagnan Baggio Emerenciano ${ }^{1}$, Roberto Tuyoshi Hosokawa ${ }^{1}$ \\ 1Eng. Florestal, Dr., Depto. de Ciências Florestais, UFPR, Curitiba, PR, Brasil - wendling@ufpr.br; darta@ufpr.br; \\ roberto.hosokawa@pq.cnpq.br
}

Recebido para publicação: 10/09/2008 - Aceito para publicação: 05/08/2010

\begin{abstract}
Resumo
Desenvolve-se uma metodologia traçada por um roteiro em algoritmo factível e amigável para efetivação em planilhas eletrônicas, reconhecidas como uma interface popular para cálculos. Buscase, assim, apresentar uma ferramenta útil para alunos de graduação e recém-graduados em engenharia florestal, ou engenheiros mais experientes que ainda não dominem a técnica, para ajuste de um modelo de função densidade de probabilidade, com o objetivo de descrever a estrutura da distribuição diamétrica de populações florestais. O modelo adotado é o da função de Weibull, e o método de ajuste é o do percentis, com simulações comparadas por teste de aderência de Kolmogorov-Smirnov. A eficiência do método apresentado é testada por comparação a outro método alternativo.

Palavras-chave: Manejo florestal; florestas - modelos matemáticos; florestas - simulação por computador.
\end{abstract}

\begin{abstract}
Weibull diameter distribution function adjusts for electronic spreadsheet. This research develops a methodology based on easy and friendly algorithm for spreadsheets, a well known interface for calculus. It aims to present a helpful tool for forestry students, as well as for newly or experienced engineers who haven't already known adjustment techniques for a density function model of probability, which is useful into diametric distribution structure descriptions of forest population. It has Weibull's function as main model, percentile as adjustment method, and comparing simulations by Kolmogorov-Smirnov goodness-of-fit test. Efficiency of the presented method was tested by comparison to another method.

Keywords: Forest management; forest - mathematical models; forest - computer simulator.
\end{abstract}

\section{INTRODUÇÃO}

O emprego de modelos de distribuição diamétrica é fundamental para a construção de quadros de produção florestal. $\mathrm{O}$ ajuste desses modelos requer um processo iterativo, não disponível automaticamente em pacotes computacionais, apropriado para solução através de uma linguagem de programação para computador. Devido ao fato de o uso dessas linguagens ainda não ser de domínio comum entre os florestais, procura-se neste trabalho apresentar um roteiro em algoritmo factível e amigável para efetivação em planilhas eletrônicas, reconhecidas como uma interface popular para cálculos. Busca-se, assim, apresentar uma ferramenta útil para alunos de graduação e recém-graduados em engenharia florestal, ou mesmo para engenheiros mais experientes que ainda não dominem a técnica, para ajuste de um modelo de função densidade de probabilidade. Para aqueles que já se aventuram em linguagens de programação, espera-se que o algoritmo possa também auxiliar em seus propósitos.

Após a apresentação do algoritmo com o roteiro e montagem da planilha, em que se constrói um simulador para escolha da melhor opção de apresentação da função, conduz-se a confrontação do método apresentado com um método alternativo de ajuste da função por regressão não linear, com o intuito de testar a eficiência do primeiro.

Objetiva-se, assim, além de apresentar uma metodologia de fácil aplicação para ajuste de uma função densidade de probabilidade e que seja precisa na descrição da estrutura da distribuição diamétrica do povoamento florestal, viabilizar o método dos percentis como instrumento plausível para uso em prognose ou projeção da produção florestal. 


\section{REVISÃO DE LITERATURA}

\section{Conceituação teórica}

Segundo Guidorizzi (2001), sendo $f$ uma função definida para todo $x$ real e integrável em todo intervalo $[a, b]$, com $a$ e $b$ reais e $a<b$, diz-se que $f$ é uma função densidade de probabilidade se

$$
\text { (1) } f(x) \geq 0 \text { para todo } x \text { e (2) } \int_{-\infty}^{+\infty} f(x) d x=1 \text {. }
$$

Sendo $X$ uma variável aleatória e $f$ uma função densidade de probabilidade, diz-se que a variável aleatória $X$ tem densidade de probabilidade $f$ se a probabilidade de $X$ pertencer ao intervalo $[a, b]$, com $a<b$ quaisquer, para

$$
\begin{aligned}
a=-\infty \text { ou } b=+\infty, \text { for dada por } P(a<X<b)=\int_{a}^{b} f(x) d x, \text { ou então, respectivamente } \\
\quad P(-\infty<X<b)=P(X<b)=\int_{-\infty}^{b} f(x) d x \text { ou } P(a<X<+\infty)=P(X<a)=\int_{a}^{+\infty} f(x) d x .
\end{aligned}
$$

\section{Função densidade de probabilidade no setor florestal}

Historicamente, na Engenharia Florestal, sempre se buscou uma maneira para descrever a estrutura da distribuição diamétrica das populações florestais, dados esses caracterizados como variável aleatória em ocorrência de distribuição contínua. Na literatura, citam-se muitos modelos definidos como funções densidade de probabilidade, sendo os principais os de Weibull, Beta, Gamma, Log-normal, Normal e SB/SBB de Johnson.

Opta-se aqui, nesta metodologia, pelo modelo de Weibull, pela sua flexibilidade para assumir diferentes formas e assimetrias, condição oportuna para o propósito de se aplicar ajuste de dados diversos em diferentes locais. Essa versatilidade levou Weibull a se tornar o mais popular dos modelos de distribuição para dados florestais.

Neste trabalho, adota-se o método dos percentis para ajuste da função Weibull. Constatam-se na literatura, nos últimos anos, poucos trabalhos em que se emprega esse método. Dentre eles, Maltamo; Kangas; Uuttera (2000) se utilizam dos percentis para comparar características de florestas com grande variedade nas distribuições diamétricas, entre povoamentos desbastados e sem desbaste de Scots pine, Pinus sylvestris L., na Finlândia. Ou, ainda, no estado da Bahia, Soares et al. (2007) empregam percentis, tomados a 50 e $75 \%$ da distribuição diamétrica em Eucalyptus sp., e as alturas correspondentes a essas posições, em modelos para realizar prognose em produção volumétrica.

Wendling (2007), ao processar o inventário florestal das parcelas de campo, faz estratégico cálculo de teste de aderência para a função de distribuição de frequências Weibull, pelo método dos percentis, ao escolher a melhor apresentação para o seu parâmetro $a$, por parcela, e depois por classe de sítio. Com isso, fornece subsídios ao seu simulador Dínamo, em que o modelo é peça fundamental na criação de cenários em quadros de produção florestal. Os cálculos são feitos por processamento em pacotes de programas para computador, compilados nas linguagens Visual FoxPro versão 8, da Microsoft Corporation, e Visual $C++$, de domínio público.

\section{MATERIAL E MÉTODOS}

\section{Formulação da metodologia}

O modelo da função de distribuição Weibull, com três parâmetros, tem a função densidade de probabilidade (fdp) expressa como:

$$
f(x)=\left\{\begin{array}{l}
\frac{c}{b}\left(\frac{x-a}{b}\right)^{c-1} e^{-\left(\frac{x-a}{b}\right)^{c},}, a \leq x<\infty, a \geq 0, \quad b>0, \quad c>0 \\
0 \longrightarrow \text { para outros casos }
\end{array}\right.
$$

Em que: $a=$ parâmetro de locação, no início da curva;

$b=$ parâmetro de escala da curva;

$c=$ parâmetro de forma, que define o tipo de curva;

$x=$ variável da distribuição, diâmetro. 
A média e a variância são expressas como:

média: $E(x)=a+b \Gamma\left(1+\frac{1}{c}\right) \quad$ variância: $V(x)=b^{2} \Gamma\left(1+\frac{2}{c}\right)-\Gamma^{2}\left(1+\frac{1}{c}\right)$

Em que: $\Gamma=$ função gama.

Para a estimativa dos parâmetros da fdp de Weibull, existem os métodos da máxima verossimilhança, dos momentos e dos percentis. Quando se conhece a verdadeira distribuição para uma dada população, qualquer método desses pode ser utilizado para se estimar a função, pois eles são equivalentes quanto à eficiência (BARRA et al., 2004). Porém se o objetivo for o de se obter um modelo de distribuição para simulação da produção florestal, em que a distribuição será estimada em cenários de prognose ou projeção e não se conhece a verdadeira distribuição, então o método deve ser aquele que gere menor dependência de funções auxiliares com projeções heterodoxas, tais como funções para projetar erro ou coeficiente de variação em diâmetro, ou $\mathrm{d}_{\mathrm{g}}$ (diâmetro médio), ou $\mathrm{d}_{\mathrm{m}}$ (diâmetro médio aritmético), ou, ainda consequentemente, expressões tipo $d_{g}-d_{m}$, 'soluções' usualmente praticadas, mas cujas validações estatísticas são discutíveis (WENDLING, 2007).

O método mais simples, claro e independente para estimar os parâmetros da fdp de Weibull naqueles cenários de simulação é o dos percentis, pois necessita apenas de funções auxiliares para estimar os dois diâmetros percentis, $\mathrm{dp}_{1}$ e $\mathrm{dp}_{2}$, com segurança similar às funções para estimar os limites diamétricos, $\mathrm{d}_{\min } \mathrm{e}$ $\mathrm{d}_{\max }$, que também devem ser usadas nesta ou em quaisquer das outras hipóteses de método.

Tabela 1. Modelos auxiliares para projeção da função de distribuição Weibull.

Table 1. Auxiliary models for Weibull distribution function projections.

\begin{tabular}{ll}
\hline Número & Função \\
\hline 1 & $\mathrm{~d}_{\max }=b_{0}+b_{1}$ idade $+b_{2}$ idade $^{2}+b_{3} \mathrm{~N}+b_{4} \mathrm{~N}^{2}+b_{5} \mathrm{~h}_{\mathrm{dom}}+b_{6} \mathrm{~h}_{\mathrm{dom}}^{2}+b_{7} \mathrm{G}+b_{8} \mathrm{G}^{2}$ \\
2 & $\mathrm{~d}_{\min }=b_{0}+b_{1} \mathrm{idade}_{+} b_{2} \mathrm{idade}^{2}+b_{3} \mathrm{~N}+b_{4} \mathrm{~N}^{2}+b_{5} \mathrm{~h}_{\mathrm{dom}}+b_{6} \mathrm{~h}_{\mathrm{dom}}^{2}+b_{7} \mathrm{G}+b_{8} \mathrm{G}^{2}+b_{9} \mathrm{~d}_{\max }+b_{10} \mathrm{~d}_{\max }^{2}$ \\
3 & $\mathrm{dp}_{1}=b_{0}+b_{1}$ idade $_{+} b_{2}$ idade $^{2}+b_{3} \mathrm{~N}+b_{4} \mathrm{~N}^{2}+b_{5} \mathrm{~h}_{\mathrm{dom}}+b_{6} \mathrm{~h}_{\mathrm{dom}}^{2}+b_{7} \mathrm{G}+b_{8} \mathrm{G}^{2}+b_{9} \mathrm{~d}_{\max }+b_{10} \mathrm{~d}_{\text {max }}^{2}$ \\
4 & $\mathrm{dp}_{2}=b_{0}+b_{1}$ idade $+b_{2}$ idade $^{2}+b_{3} \mathrm{~N}+b_{4} \mathrm{~N}^{2}+b_{5} \mathrm{~h}_{\mathrm{dom}}+b_{6} \mathrm{~h}_{\mathrm{dom}}^{2}+b_{7} \mathrm{G}+b_{8} \mathrm{G}^{2}+b_{9} \mathrm{~d}_{\max }+b_{10} \mathrm{~d}_{\max }^{2}$ \\
\hline
\end{tabular}

Fonte: Wendling (2007).

$\mathrm{d}_{\max }, \mathrm{d}_{\min }=\mathrm{DAP}$ (diâmetros à altura do peito, em $\mathrm{cm}$ ) máximo e mínimo do povoamento. dp $\mathrm{p}_{1}$ e dp $\mathrm{d}_{2}=\mathrm{DAP}$ percentis a $24 \%$ e a $93 \%$ na distribuição de frequências. Idade do povoamento $=\mathrm{em}$ anos, em decimais. $\mathrm{N}=$ número de árvores por hectare do povoamento. $\mathrm{h}_{\text {dom }}=$ média das alturas das 100 árvores de maior diâmetro do povoamento, em $\mathrm{m}$. $\mathrm{G}=$ área basal do povoamento, em $\mathrm{m}^{2}$. $b_{0}, b_{1} \ldots b_{10}=$ coeficientes a estimar.

Para ilustrar as vantagens do método dos percentis, pode-se citar Wendling (2007) que, para viabilizar a projeção da função de distribuição em diâmetros no simulador da produção florestal Dínamo, constrói um sistema prático e viável de equações auxiliares, que permitem o ajuste da função de Weibull em diversas idades. Os modelos, definidos conforme a tabela 1, são ajustados separadamente por grupo de parcelas de cada classe de sítio e, ainda, somente para aquelas parcelas de povoamentos que tenham atingido, em seu desenvolvimento, o estágio de "povoamento em densidade completa".

Como demonstrado por Scolforo (1998), no método dos percentis os parâmetros $a, b$ e $c$ se relacionam nas fórmulas

$$
\begin{gathered}
a=x-\frac{b}{n^{\frac{1}{c}}} \Gamma\left(1+\frac{1}{c}\right) \\
c=\frac{\ln [-\ln (1-\mathrm{p} 1) /-\ln (1-\mathrm{p} 2)]}{\ln \left[\left(x_{\mathrm{p} 1}-a\right) /\left(x_{\mathrm{p} 2}-a\right)\right]} \\
b=\frac{x_{\mathrm{p} 1}-a}{(-\ln (1-\mathrm{p} 1))^{\frac{1}{c}}}
\end{gathered}
$$

Em que: $x=$ diâmetro da parcela;

$n=$ número de árvores da parcela;

$\Gamma=$ função gama;

$\mathrm{p} 1$ e p2 = percentis de $\mathrm{n}$;

$x_{\mathrm{p} 1}$ e $x_{\mathrm{p} 2}=\mathrm{dp}_{1}$ e $\mathrm{dp}_{2}=$ diâmetros para $\mathrm{p} 1$ e p2. 
A aplicação de todas essas fórmulas simultaneamente para se estimar os parâmetros $a, b$ e $c$ é inviável, pois em todas elas aparece um ou outro dos demais parâmetros, como variável independente. Como solução, deve-se começar a estimar $b$ e $c$, simulando-se separadamente o parâmetro $a$, atribuindolhe um percentual do diâmetro mínimo, que pode ser $0,0 \mathrm{~d}_{\min }, 0,1 \mathrm{~d}_{\min }, 0,2 \mathrm{~d}_{\min }, 0,3 \mathrm{~d}_{\min }, 0,4 \mathrm{~d}_{\min }, 0,5 \mathrm{~d}_{\min }$, $0,6 \mathrm{~d}_{\min }, 0,7 \mathrm{~d}_{\min }, 0,8 \mathrm{~d}_{\min }, 0,9 \mathrm{~d}_{\min }, 1,0 \mathrm{~d}_{\min }$. Assim como em Wendling (2007), para cada valor de $a$ calculam-se os valores correspondentes para $b$ e $c$, em cada parcela, juntamente com o teste de aderência Kolmogorov-Smirnov para cada distribuição ajustada. Os valores calculados para a melhor distribuição por parcela, escolhida pelo teste, no final são indicados para aplicação em nova planilha, para posterior levantamento da melhor proposta de $a$ por classe de sítio.

O teste de aderência Kolmogorov-Smirnov compara a frequência acumulativa estimada com a frequência acumulativa observada, em cada classe de diâmetro entre $d_{\min }$ e $d_{\max }$. O ponto de maior divergência entre as duas distribuições é o valor $D$ de Kolmogorov-Smirnov. Esse valor é comparado com o valor tabelar que, segundo Haldar; Mahadevan (2000), é calculado por

$$
d_{1 \%}^{n}=\frac{1,63}{\sqrt{n-1}}, \text { ou } d_{1 \%}^{n}=\frac{1,63}{\sqrt{n}} \text { para } \mathrm{n}>=50,
$$

em que $\mathrm{P}(D>=d)=\alpha$, para $\alpha=0,01$.

Se o valor $D$ for menor do que o tabelar $d$, estabelecido para o número total de árvores da parcela, $n$, verifica-se que o valor de maior divergência não é significativo, o que indica que nesse caso há aderência dos dados à distribuição Weibull, com 99\% de probabilidade de acerto.

Glade (1986) cita, em recomendação de Bailey (1973), como ideais os percentis de 0,24n e 0,93n, para p1 e p2, valores esses também adotados em Wendling (2007) e neste trabalho.

\section{Material da metodologia}

Os dados de campo apresentados são da espécie Pinus taeda L. e foram coletados pela empresa International Paper do Brasil, em povoamentos florestais de sua propriedade, no município de Arapoti, estado do Paraná. São de parcela medida em área de $600 \mathrm{~m}^{2}$ (20 x $\left.30 \mathrm{~m}\right)$, com idade de 13,1 anos. Para os dados das reaplicações, utilizaram-se parcelas de 5,4 a 21 anos. Por se tratar de uma metodologia, os dados servem apenas como um exemplo de aplicação.

Devido às numerosas sequências de operações e procedimentos para aplicação da metodologia em planilhas eletrônicas, a narração segue roteiro esquematizado em algoritmo descrito na tabela 2 , do passo 1 ao passo 21, e ilustrado nas figuras 1 a 5. Os procedimentos estão caracterizados em comandos peculiares a quaisquer programas de planilha eletrônica existentes no mercado, tais como StarOffice da Sun Microsystems disponível em versões livre ou proprietária -, OpenOffice - em versão livre para sistemas Linux - e Excel, da Microsoft Corporation - em versão proprietária. As definições desses comandos podem ser obtidas nos manuais dos aplicativos, tais como o manual de ajuda off-line do Excel em Microsoft Corporation (2007).

\section{Aplicação da metodologia}

O início do algoritmo consiste em colocar (colar) os dados de DAP da parcela, conforme o passo 1 da tabela 2, em coluna a partir da célula B4, tal como se pode visualizar na figura 1. Essa planilha inicial, ainda vazia, apresenta os cabeçalhos previamente preparados e valores prefixados para os percentis $\mathrm{p} 1 \mathrm{e} \mathrm{p} 2$. No passo 2 do algoritmo, faz-se a classificação dos dados de DAP em ordem crescente. Observe-se que no Excel é necessário escolher a coluna dos dados, para que se efetive a operação. Depois, faz-se a numeração dos dados de DAP, a partir da primeira árvore existente, conforme passo 3 da tabela 2 e figura 2. Para a parcela usada como exemplo, a célula que corresponde à primeira árvore é B27, então a numeração se inicia em A27. Os dados que aparecem zerados são das árvores mortas ou falhas e, por isso, não têm medição de DAP. A última árvore existente é a da célula B137.

No passo 4 e nas linhas superiores da planilha da figura 2, procede-se aos cálculos das variáveis de configuração para as fórmulas dos parâmetros da função de Weibull: $\mathrm{n}\left(\mathrm{n}^{\circ}\right.$ de árvores da parcela); $\mathrm{n}^{\circ} \mathrm{s}$ das árvores que correspondem às posições percentis 1 e 2, na lista classificada no passo 2; e os valores dos limites da distribuição diamétrica, $\mathrm{d}_{\min }$ e $\mathrm{d}_{\max }$. Esses cálculos se completam no passo 5 , ao se capturarem os valores de DAP correspondentes àquelas posições percentis, 1 e 2, apontadas nas células F5 e F6. 
Tabela 2. Algoritmo para ajuste da função de distribuição Weibull, em planilha eletrônica.

Table 2. Algorithm for Weibull distribution function fitting, by electronic spreadsheet.

\begin{tabular}{|c|c|c|c|}
\hline Passo & Operação & Endereço (células) & Procedimento \\
\hline 1 & $\begin{array}{l}\text { Colar os dados de DAP da parcela, em } \\
\text { coluna }\end{array}$ & A partir de B4 & Copiar-colar \\
\hline 2 & $\begin{array}{l}\text { Classificar em ordem crescente a coluna } \\
\text { DAP }\end{array}$ & $\begin{array}{l}\text { De A4 e B4 até a última } \\
\text { árvore }\end{array}$ & $\begin{array}{l}\text { Dados / filtrar por valor / classificar do menor para o } \\
\text { maior }\end{array}$ \\
\hline 3 & Numerar as árvores existentes & $\begin{array}{l}\text { De A27 (no caso) } \\
\text { até a última árvore }\end{array}$ & $\begin{array}{l}\text { Digitar } 1 \text { e } 2 \text { nas primeiras e } \\
\text { replicar às demais }\end{array}$ \\
\hline \multirow{5}{*}{4} & \multirow{5}{*}{ 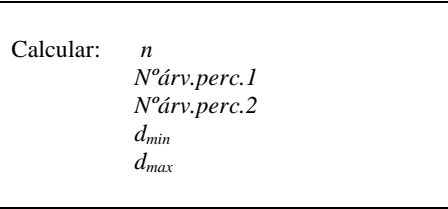 } & F4 & $=$ CONT.NÚM(B27:B137) \\
\hline & & F5 & $=\mathrm{J} 5 * \mathrm{~F} \$ 4$ \\
\hline & & F6 & $=\mathrm{J} 6 * \mathrm{~F} \$ 4$ \\
\hline & & H3 & =MÍNIMO(B27:B137) \\
\hline & & $\mathrm{H} 4$ & =MÁXIMO(B27:B137) \\
\hline \multirow{2}{*}{5} & \multirow{2}{*}{$\begin{array}{ll}\text { Estabelecer: } & d p_{1} \\
& d p_{2}\end{array}$} & H5 & =B53 (capturar na coluna DAP, seg. $N^{o}$ árv.perc. 1$)$ \\
\hline & & H6 & =B129 (idem, segundo $N^{o}$ árv.perc. 2 ) \\
\hline 6 & $\begin{array}{l}\text { Importar os valores calculados no Statistica } \\
\text { para a coluna fo (frequências observadas) }\end{array}$ & $\begin{array}{l}\text { De H10 até H34 (no } \\
\text { caso) }\end{array}$ & Copiar-colar \\
\hline \multirow{4}{*}{7} & \multirow{4}{*}{$\begin{array}{ll}\text { Calcular } & \text { centro, } \\
& \text { limite inferior e } \\
& \text { limite superior das classes }\end{array}$} & F10 & $=\mathrm{INT}(\mathrm{H} \$ 3+0,5)-1+\mathrm{D} 10$ \\
\hline & & E10 & $=\mathrm{F} 10-0,5$ \\
\hline & & G10 & $=\mathrm{F} 10+0,5$ \\
\hline & & até F34, E34 e G34 & replicar F10, E10 e G10 \\
\hline 8 & $\begin{array}{l}\text { Calcular a soma das frequências (comparar } \\
\text { com o calculado em F4) }\end{array}$ & H35 (no caso) & $=\mathrm{SOMA}(\mathrm{H} 10: \mathrm{H} 34)$ \\
\hline \multirow{3}{*}{9} & \multirow{3}{*}{$\begin{array}{l}\text { Preparar a coluna acum (frequências } \\
\text { observadas acumuladas) }\end{array}$} & $\mathrm{I} 10$ & $=\mathrm{H} 10$ \\
\hline & & I11 & $=\mathrm{H} 11+\mathrm{I} 10$ \\
\hline & & I12 até I34 & replicar I11 \\
\hline \multirow{2}{*}{10} & \multirow{2}{*}{$\begin{array}{l}\text { Calcular a coluna da distribuição } \\
\text { acumulativa observada, } S(x)\end{array}$} & $\mathrm{J} 10$ & $=\mathrm{I} 10 / \mathrm{H} \$ 35$ \\
\hline & & J11 até J34 & replicar J10 \\
\hline 11 & $\begin{array}{l}\text { Calcular valor para o parâmetro } a \text { da } \\
\text { função Weibull, primeira simulação }\end{array}$ & L5 & $=\mathrm{L} 4 * \$ \mathrm{H} 3$ \\
\hline 12 & $\begin{array}{l}\text { Calcular } \\
c=\frac{\ln [-\ln (1-\mathrm{p} 1) /-\ln (1-\mathrm{p} 2)]}{\ln \left[\left(x_{\mathrm{p} 1}-a\right) /\left(x_{\mathrm{p} 2}-a\right)\right]}\end{array}$ & L6 & $\begin{array}{l}=\mathrm{LN}(-\mathrm{LN}(1-\$ \mathrm{~J} 5) /-\mathrm{LN}(1-\$ \mathrm{~J} 6)) / \mathrm{LN}((\$ \mathrm{H} 5- \\
\mathrm{L} 5) /(\$ \mathrm{H} 6-\mathrm{L} 5))\end{array}$ \\
\hline 13 & $\begin{array}{l}\text { Calcular } \\
\qquad b=\frac{x_{\mathrm{p} 1}-a}{(-\ln (1-\mathrm{p} 1))^{\frac{1}{c}}}\end{array}$ & L7 & $=(\$ \mathrm{H} 5-\mathrm{L} 5) /-\mathrm{LN}(1-\$ \mathrm{~J} 5)^{\wedge}(1 / \mathrm{L} 6)$ \\
\hline \multirow[b]{2}{*}{14} & \multirow{2}{*}{$\begin{array}{l}\text { Calcular a coluna da distribuição } \\
\text { acumulativa estimada por Weibull } \\
F(x)=1-e^{-\left(\frac{x-a}{b}\right)^{c}}\end{array}$} & L10 & $=1-\mathrm{EXP}\left(-\left(((\$ \mathrm{G} 10-\mathrm{L} \$ 5) / \mathrm{L} \$ 7)^{\wedge} \mathrm{L} \$ 6\right)\right)$ \\
\hline & & L11 até L34 & replicar L10 \\
\hline \multirow[b]{2}{*}{15} & \multirow{2}{*}{$\begin{array}{l}\text { Calcular a coluna das divergências entre os } \\
\text { valores das colunas de distribuição } \\
\text { acumulativa observada e de distribuição } \\
\text { acumulativa estimada, }|F(x)-S(x)|\end{array}$} & M10 & $=\mathrm{ABS}(\mathrm{L} 10-\$ \mathrm{~J} 10)$ \\
\hline & & M11 até M34 & replicar M10 \\
\hline 16 & Calcular o valor de maior divergência & M36 & =MÁXIMO(M10:M34) \\
\hline \multirow{2}{*}{17} & $\begin{array}{l}\text { Colocar a fórmula da tabela de } \\
\text { Kolmogorov-Smirnov }\end{array}$ & M42 & $=1,63 /$ RAIZ $(\mathrm{H} 35)$ \\
\hline & $\begin{array}{l}\text { Comparar o valor de maior divergência } \\
\text { com o tabelar }\end{array}$ & M37 & =SE(M36>\$M42;"não aderiu";"aderiu") \\
\hline 18 & $\begin{array}{l}\text { Replicar a simulação da opção de } \\
0,0 \mathrm{~d}_{\min } \text { (feita nos passos } 11 \text { a } 17 \text { ), para as } \\
\text { simulações das demais opções de } 0,1 \mathrm{~d}_{\min } \\
\text { até } 1,0 \mathrm{~d}_{\min }\end{array}$ & $\begin{array}{l}\text { A partir de N5 e, depois, } \\
\text { a partir de P5, R5, T5, } \\
\text { V5, X5, Z5, AB5, AD5 e } \\
\text { AF5 }\end{array}$ & Marcar a área L5:M37 e replicar \\
\hline 19 & $\begin{array}{l}\text { Calcular a menor das divergências entre as } \\
11 \text { opções calculadas }\end{array}$ & M40 & $\begin{array}{l}\text { =MÍNIMO(M36;O36;Q36;S36;U36;W36;Y36; } \\
\text { AA36;AC36;AE36;AG36) }\end{array}$ \\
\hline \multirow[b]{2}{*}{20} & \multirow{2}{*}{$\begin{array}{l}\text { Assinalar (+) na coluna correspondente à } \\
\text { menor das divergências, calculada no passo } \\
19, \text { por meio de comparação }\end{array}$} & M38 & =SE(M36=\$M40;" +";"") \\
\hline & & $\begin{array}{l}\text { O38; Q38; S38; U38; } \\
\text { W38; Y38; AA38; AC38; } \\
\text { AE38; AG38 }\end{array}$ & replicar M38 \\
\hline 21 & $\begin{array}{l}\text { Capturar os valores dos parâmetros } a, b \text { e } c \\
\text { da melhor opção para a função Weibull }\end{array}$ & $\begin{array}{l}\text { Valores da opção que } \\
\text { recebeu (+) }\end{array}$ & $\begin{array}{l}\text { Copiar e colar em outra planilha, para a devida } \\
\text { aplicação }\end{array}$ \\
\hline
\end{tabular}


A próxima etapa do algoritmo é o cálculo do número de árvores por classe diamétrica. Essa tarefa pode ser mais bem executada por pacotes estatísticos conhecidos, tais como Statgraphics, da Statistical Graphics Corp., Matlab, da MathWorks Inc., $R$, da The $\mathrm{R}$ Foundation for Statistical Computing, Statistica, da StatSoft Inc., e outros.

Escolhe-se aqui o Statistica. Após serem colocados os dados de DAP da parcela na sua prancheta de cálculos, aciona-se a opção Basic Statistics/Tables, depois Frequency Tables. Deve-se garantir que a contagem das frequências ocorra com os centros de classe nos valores arredondados, por exemplo: $9,0 \mathrm{~cm}$ para a classe de 8,5 até $9,49 \mathrm{~cm}$, e assim por diante. Para tal feito, na configuração no Frequency Tables há que se observarem as redefinições: marca-se Step size $=1$, desmarca-se at minimum e altera-se para starting at 0,5 .

Então, no passo 6 e figura 2, importam-se esses valores calculados no Statistica para a coluna fo (frequências observadas). Essa tabela de classes gerada deve ser adaptada em número de linhas, de acordo com a amplitude dos dados da parcela em estudo.

No passo 7, calculam-se os valores dos centros e limites das classes. Nessa tabela de classes, nota-se que os seus limites superiores de classe estão em intervalos abertos, combinando com os limites inferiores das classes seguintes em intervalos fechados. Essa notação pode ser explicada, por motivo da característica da aplicação da fórmula, mais adiante, no passo 14. Ao se calcularem os valores da distribuição acumulativa estimada por Weibull, pela fórmula da integral da função, essa fórmula é empregada a partir do valor em cada limite superior dessa tabela, em descontos do valor total, o que gera cálculos à esquerda desses pontos da curva. Ou seja, os cálculos são feitos, pela curva da integração, para valores menores do que os valores desses limites superiores, o que resulta em intervalos abertos para esses limites. Então a interpretação dos cálculos para a classe 9,0, por exemplo, fica circunscrita ao intervalo [ $8,5 \leq 9,0<9,5]$, o que equivale a $[8,5 \leq 9,0 \leq 9,49]$, e assim para o restante da tabela de classes.

Somam-se as frequências, no passo 8 . No passo 9 , faz-se a preparação da coluna com as frequências observadas acumuladas. No passo 10, organiza-se a coluna da distribuição acumulativa observada, $S(x)$, que divide cada elemento da coluna do passo 9 pelo valor da soma das frequências.

Para as etapas finais do algoritmo da tabela 2, ilustradas em planilha nas figuras 3 a 5 , os passos 11 a 17 calculam a primeira simulação em busca do melhor ajuste do modelo de distribuição, sendo que ao parâmetro $a$ da função Weibull é atribuído o valor inicial de $0,0 \mathrm{~d}_{\min }$. Para esse valor fixado no passo 11, calculam-se, nos passos 12 e 13, os valores para os parâmetros $c$ e $b$, por suas fórmulas lá expostas.

No passo 14 são calculados os valores da distribuição acumulativa estimada por Weibull, $F(x)$, pela fórmula da integral da função, mostrada na tabela. A diferença, em valores absolutos, entre essa coluna gerada e a coluna calculada no passo 10 define a coluna das divergências entre os valores das colunas de distribuição acumulativa observada e de distribuição acumulativa estimada, $|F(x)-S(x)|$, tal como se processa no passo 15 .

Para completar a primeira simulação, compete ao passo 16 calcular o valor de maior divergência entre os valores da coluna $|F(x)-S(x)|$. Ao passo 17, finalmente, cabe fazer a comparação desse valor com o tabelar de Kolmogorov-Smirnov, formulado na célula M42 como ${ }_{{ }_{1 \%}^{n}}^{n}=\frac{1,63}{\sqrt{n}}$.

Na busca da melhor apresentação da função Weibull, o passo 18 da tabela 2 trata de replicar o processo da primeira simulação, feita para a opção de $0,0 \mathrm{~d}_{\text {min }}$, para as demais opções, em que ao parâmetro $a$ da função Weibull são atribuídos, respectivamente, valores de $0,1 \mathrm{~d}_{\min }, 0,2 \mathrm{~d}_{\min }, 0,3 \mathrm{~d}_{\min }$, $0,4 \mathrm{~d}_{\min }, 0,5 \mathrm{~d}_{\min }, 0,6 \mathrm{~d}_{\min }, 0,7 \mathrm{~d}_{\min }, 0,8 \mathrm{~d}_{\min }, 0,9 \mathrm{~d}_{\min }$ e $1,0 \mathrm{~d}_{\min }$. O termo 'replicar', em planilhas eletrônicas, significa copiar as células com as fórmulas para outro endereço, de forma similar, porém adaptadamente, com a variação de alguma variável, no caso os valores calculados para o parâmetro $a$. Têm-se, então, conforme ilustrado nas figuras 4 e 5 , as demais simulações que completam o método proposto de ajuste da função.

No passo 19, calcula-se a menor das divergências, dentre os valores calculados como maior divergência em cada uma das 11 opções simuladas. O passo 20 busca e assinala na linha 40 da planilha, com o símbolo (+), a coluna da simulação correspondente à menor das divergências, por comparação ao valor levantado no passo 19.

No último passo, o 21, faz-se a coleta manual dos valores da opção que recebeu o símbolo (+). Ou seja, capturam-se os valores dos parâmetros $a, b$ e $c$ dessa melhor opção para a função Weibull, para a devida aplicação em outra planilha, com objetivos do manejo florestal. Encerra-se, assim, o processo de simulação do algoritmo. 


\begin{tabular}{|c|c|c|c|c|c|c|c|c|c|c|c|c|c|}
\hline 1 & A & $\mathrm{C}$ & $\mathrm{D}$ & $\mathrm{E}$ & $\mathrm{F}$ & G & $\mathrm{H}$ & I & $\mathrm{J}$ & $\mathrm{K}$ & $\mathrm{L}$ & M & $\mathrm{N}$ \\
\hline 2 & & & & & & & & & & & & & \\
\hline 3 & $\underline{\mathbf{n}^{\mathbf{0}}}$ & $\underline{\mathbf{D A P}(\mathbf{c m})}$ & & & & $d_{\min }=$ & & & & \multicolumn{4}{|c|}{ Parâmetros da função Weibull } \\
\hline 4 & & 24,8 & & $\mathbf{n}=$ & & dmáx= & & & \multicolumn{2}{|c|}{$\% \mathbf{d}_{\min }=$} & $\mathbf{0}$ & & $\mathbf{0 , 1}$ \\
\hline 5 & & 18,1 & \multicolumn{2}{|c|}{$\mathbf{N}^{0}$.árv.perc.1 = } & & $d_{1}=$ & & $\mathbf{p} 1=($ & 0,24 & $a=$ & & & \\
\hline 6 & & 18,8 & \multicolumn{2}{|c|}{$\mathrm{N}^{0}$.árv.perc. $2=$} & & $d_{2}=$ & & $\mathbf{p} 2=($ & 0,93 & $c=$ & & & \\
\hline 7 & & 15,3 & & & & & & & & $b=$ & & & \\
\hline 8 & & 15,0 & \multicolumn{5}{|c|}{ C L A S S E S DAP (cm) } & & & & & & \\
\hline 9 & & 8,9 & $\mathbf{n}^{\mathbf{o}}$ & lim.inf $\leq$ & centro & lim.sup & fo & acum & $S(x)$ & & & & \\
\hline 10 & & 19,1 & $\mathbf{1}$ & & & & & & & & & & \\
\hline 11 & & 15,0 & 2 & & & & & & & & & & \\
\hline 12 & & 0,0 & 3 & & & & & & & & & & \\
\hline 13 & & 23,6 & 4 & & & & & & & & & & \\
\hline 14 & & 0,0 & 5 & & & & & & & & & & \\
\hline 15 & & 11,1 & 6 & & & & & & & & & & \\
\hline 16 & & 27,4 & 7 & & & & & & & & & & \\
\hline 17 & & 14,0 & 8 & & & & & & & & & & \\
\hline 18 & & 21,6 & 9 & & & & & & & & & & \\
\hline 19 & & 0,0 & 10 & & & & & & & & & & \\
\hline 20 & & 16,2 & 11 & & & & & & & & & & \\
\hline 21 & & 19,4 & 12 & & & & & & & & & & \\
\hline 22 & & 14,0 & 13 & & & & & & & & & & \\
\hline 23 & & 18,1 & 14 & & & & & & & & & & \\
\hline 24 & & 23,9 & 15 & & & & & & & & & & \\
\hline 25 & & 32,8 & 16 & & & & & & & & & & \\
\hline 26 & & 0,0 & 17 & & & & & & & & & & \\
\hline 27 & & 0,0 & 18 & & & & & & & & & & \\
\hline 28 & & 16,6 & 19 & & & & & & & & & & \\
\hline 29 & & 19,1 & 20 & & & & & & & & & & \\
\hline 30 & & 13,1 & 21 & & & & & & & & & & \\
\hline 31 & & 20,7 & 22 & & & & & & & & & & \\
\hline 32 & & 14,0 & 23 & & & & & & & & & & \\
\hline 33 & & 25,8 & 24 & & & & & & & & & & \\
\hline 34 & & 18,5 & 25 & & & & & & & & & & \\
\hline 35 & & 18,1 & & & & $\mathbf{n}=$ & & & & & & & \\
\hline 36 & & 15,3 & & & & & & & & & & & \\
\hline 37 & & 19,4 & & & & & & & & & & & \\
\hline 38 & & 17,5 & & & & & & & & & & & \\
\hline 39 & & 22,0 & & & & & & & & & & & \\
\hline 40 & & 13,7 & & & & & & & $4 \times$ & & & & \\
\hline 41 & & 17,5 & & & & Cabe & çall & 1os seg & guem ate & & & & \\
\hline 42 & & 25,1 & & & & & & $0 \mathrm{~d}_{\mathrm{min}}$ & & & & & \\
\hline 43 & & 18,5 & & & & & & & & & & & \\
\hline 44 & & 16,9 & & & & & & & & & & & \\
\hline 45 & & 18,5 & & \multirow{2}{*}{\multicolumn{3}{|c|}{$\begin{array}{l}\text { Dados seguem a té a } \\
\text { última árvore }\end{array}$}} & & & & & & & \\
\hline 46 & & 26,4 & & & & & & & & & & & \\
\hline 47 & & 15,3 & & & & & & & & & & & \\
\hline 48 & & 23,9 & & & & & & & & & & & \\
\hline 49 & & 16,9 & & & & & & & & & & & \\
\hline 50 & & 0,0 & & & & & & & & & & & \\
\hline
\end{tabular}

Figura 1. Planilha inicial, com entrada dos dados de campo e cabeçalhos iniciais.

Figure 1. Initial spreadsheet, with data incomes and initial headlines. 


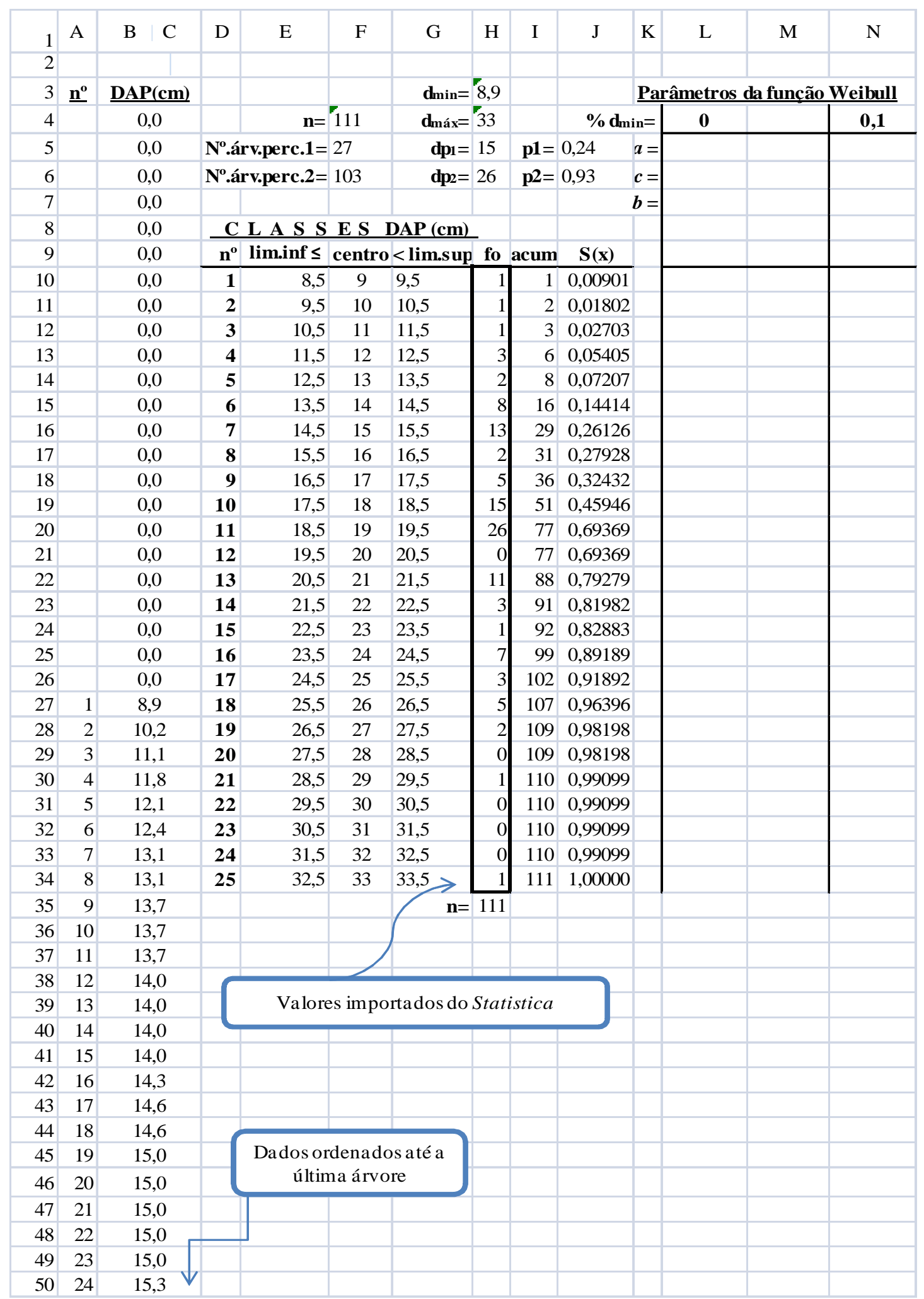

Figura 2. Planilha com cálculos iniciais.

Figure 2. Spreadsheet with initial calculations. 


\begin{tabular}{|c|c|c|c|c|c|c|c|c|c|c|c|c|c|}
\hline 1 & A & $\mathrm{B} \mid \mathrm{C}$ & $\mathrm{D}$ & $\mathrm{E}$ & $\mathrm{F}$ & G & $\mathrm{H}$ & I & $\mathbf{J}$ & $\mathrm{K}$ & $\mathrm{L}$ & M & $\mathrm{N}$ \\
\hline 2 & & & & & & & & & & & & & \\
\hline 3 & $\underline{\mathbf{n}}^{\mathbf{0}}$ & $\underline{\operatorname{DAP}(\mathbf{c m})}$ & & & & $d_{\min }=$ & 8,9 & & & \multicolumn{4}{|c|}{ Parâmetros da função Weibull } \\
\hline 4 & & 0,0 & & $\mathbf{n}=$ & 111 & dmáx $=$ & 33 & & \multicolumn{2}{|c|}{$\% \mathrm{~d}_{\min }=$} & $\mathbf{0}$ & & $\mathbf{0 , 1}$ \\
\hline 5 & & 0,0 & \multicolumn{3}{|c|}{$\mathbf{N}^{0}$.árv.perc. $1=27$} & $d p_{1}=$ & 15 & $\mathbf{p} 1=$ & 0,24 & $a=$ & 0 & & \\
\hline 6 & & 0,0 & \multirow{2}{*}{\multicolumn{3}{|c|}{$\mathbf{N}^{\mathbf{0}}$.árv.perc. $\mathbf{2}=103$}} & $\mathrm{dp} 2=$ & 26 & $\mathrm{p} 2=$ & 0,93 & $c=$ & 4,34638825 & & \\
\hline 7 & & 0,0 & & & & & & & & $b=$ & 20,6011945 & & \\
\hline 8 & & 0,0 & \multicolumn{5}{|c|}{ 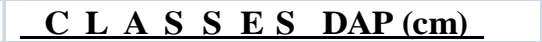 } & & & & & & \\
\hline 9 & & 0,0 & $\mathbf{n}^{\mathbf{o}}$ & $\lim . i n f \leq$ & centro & $<$ lim.sup & fo: & acum & $\mathbf{S}(\mathbf{x})$ & & $\mathbf{F}(\mathbf{x})$ & $|\mathbf{F}(\mathbf{x})-\mathbf{S}(\mathbf{x})|$ & \\
\hline 10 & & 0,0 & $\mathbf{1}$ & 8,5 & 9 & 9,5 & 1 & 1 & 0,00901 & & 0,03399 & 0,02498 & \\
\hline 11 & & 0,0 & 2 & 9,5 & 10 & 10,5 & 1 & 2 & 0,01802 & & 0,05203 & 0,03401 & \\
\hline 12 & & 0,0 & 3 & 10,5 & 11 & 11,5 & 1 & 3 & 0,02703 & & 0,07628 & 0,04925 & \\
\hline 13 & & 0,0 & 4 & 11,5 & 12 & 12,5 & 3 & 6 & 0,05405 & & 0,10774 & 0,05369 & \\
\hline 14 & & 0,0 & 5 & 12,5 & 13 & 13,5 & 2 & 8 & 0,07207 & & 0,14725 & 0,07518 & \\
\hline 15 & & 0,0 & 6 & 13,5 & 14 & 14,5 & 8 & 16 & 0,14414 & & 0,19532 & 0,05117 & \\
\hline 16 & & 0,0 & 7 & 14,5 & 15 & 15,5 & 13 & 29 & 0,26126 & & 0,25202 & 0,00925 & \\
\hline 17 & & 0,0 & 8 & 15,5 & 16 & 16,5 & 2 & 31 & 0,27928 & & 0,31685 & 0,03757 & \\
\hline 18 & & 0,0 & 9 & 16,5 & 17 & 17,5 & 5 & 36 & 0,32432 & & 0,38865 & 0,06432 & \\
\hline 19 & & 0,0 & 10 & 17,5 & 18 & 18,5 & 15 & 51 & 0,45946 & & 0,46555 & 0,00609 & \\
\hline 20 & & 0,0 & 11 & 18,5 & 19 & 19,5 & 26 & 77 & 0,69369 & & 0,54506 & 0,14863 & \\
\hline 21 & & 0,0 & 12 & 19,5 & 20 & 20,5 & 0 & 77 & 0,69369 & & 0,62425 & 0,06945 & \\
\hline 22 & & 0,0 & 13 & 20,5 & 21 & 21,5 & 11 & 88 & 0,79279 & & 0,69999 & 0,09280 & \\
\hline 23 & & 0,0 & 14 & 21,5 & 22 & 22,5 & 3 & 91 & 0,81982 & & 0,76938 & 0,05044 & \\
\hline 24 & & 0,0 & 15 & 22,5 & 23 & 23,5 & 1 & 92 & 0,82883 & & 0,83004 & 0,00121 & \\
\hline 25 & & 0,0 & 16 & 23,5 & 24 & 24,5 & 7 & 99 & 0,89189 & & 0,88046 & 0,01144 & \\
\hline 26 & & 0,0 & 17 & 24,5 & 25 & 25,5 & 3 & 102 & 0,91892 & & 0,92014 & 0,00122 & \\
\hline 27 & 1 & 8,9 & 18 & 25,5 & 26 & 26,5 & 5 & 107 & 0,96396 & & 0,94958 & 0,01438 & \\
\hline 28 & 2 & 10,2 & 19 & 26,5 & 27 & 27,5 & 2 & 109 & 0,98198 & & 0,97008 & 0,01190 & \\
\hline 29 & 3 & 11,1 & 20 & 27,5 & 28 & 28,5 & 0 & 109 & 0,98198 & & 0,98340 & 0,00142 & \\
\hline 30 & 4 & 11,8 & 21 & 28,5 & 29 & 29,5 & 1 & 110 & 0,99099 & & 0,99145 & 0,00046 & \\
\hline 31 & 5 & 12,1 & 22 & 29,5 & 30 & 30,5 & 0 & 110 & 0,99099 & & 0,99593 & 0,00494 & \\
\hline 32 & 6 & 12,4 & 23 & 30,5 & 31 & 31,5 & 0 & 110 & 0,99099 & & 0,99822 & 0,00723 & \\
\hline 33 & 7 & 13,1 & 24 & 31,5 & 32 & 32,5 & 0 & 110 & 0,99099 & & 0,99929 & 0,00830 & \\
\hline 34 & 8 & 13,1 & 25 & 32,5 & 33 & 33,5 & 1 & 111 & 1,00000 & & 0,99975 & 0,00025 & \\
\hline 35 & 9 & 13,7 & & & & $\mathbf{n}=$ & 111 & & & & & & \\
\hline 36 & 10 & 13,7 & & & & & & & Maior d & iver: & gência ==> & 0,148630 & \\
\hline 37 & 11 & 13,7 & & & & & & & Kolmog & orov & -Smirnov => & aderiu & \\
\hline 38 & 12 & 14,0 & & & & & & & & & & & \\
\hline 39 & 13 & 14,0 & & & & & & & & & & & \\
\hline 40 & 14 & 14,0 & & & & & & & Melhor & Ade & rência $==>$ & 0,103036 & $(+)$ \\
\hline 41 & 15 & 14,0 & & & & & & & & & & & \\
\hline 42 & 16 & 14,3 & & & & & & & Valo & r tal & pelar $==>$ & 0,154713 & \\
\hline 43 & 17 & 14,6 & & & & & & & & & & & \\
\hline
\end{tabular}

Figura 3. Planilha com cálculos da primeira simulação.

Figure 3. Spreadsheet with the first simulation calculation. 


\begin{tabular}{|c|c|c|c|c|c|c|c|c|c|}
\hline $\mathrm{N}$ & $\mathrm{O}$ & $\mathrm{P}$ & Q & $\mathrm{R}$ & $\mathrm{S}$ & $\mathrm{T}$ & $\mathrm{U}$ & V & $\mathrm{W}$ \\
\hline & & & & \multicolumn{4}{|c|}{ Replicações seguem até $1,0 \mathrm{~d}_{\min }$} & & \\
\hline \multicolumn{10}{|l|}{ Weibull } \\
\hline $\mathbf{0 , 1}$ & & $\mathbf{0 , 2}$ & & $\mathbf{0 , 3}$ & & $\mathbf{0 , 4}$ & & $\mathbf{0 , 5}$ & \\
\hline 0,89 & & 1,78 & & 2,67 & & 3,56 & & 4,45 & \\
\hline 4,149255 & & 3,951655 & & 3,75351 & & 3,554727 & & 3,355188 & \\
\hline 19,67901 & & 18,75356 & & 17,82428 & & 16,89052 & & 15,95143 & \\
\hline $\mathbf{F}(\mathbf{x})$ & $|\mathbf{F}(\mathbf{x})-\mathbf{S}(\mathbf{x})|$ & $\mathbf{F}(\mathbf{x})$ & $\mid \mathbf{F}(\mathbf{x})-\mathbf{S}(\mathbf{x})$ & $\mathbf{F}(\mathbf{x})$ & $\mid \mathbf{F}(\mathbf{x})-\mathbf{S}(\mathbf{x})$ & $\mathbf{F}(\mathbf{x})$ & $|\mathbf{F}(\mathbf{x})-\mathbf{S}(\mathbf{x})|$ & $\mathbf{F}(\mathbf{x})$ & $|\mathbf{F}(\mathbf{x})-\mathbf{S}(\mathbf{x})|$ \\
\hline 0,03187 & 0,02286 & 0,02953 & 0,02052 & 0,02694 & 0,01793 & 0,02406 & 0,01506 & 0,02087 & 0,01186 \\
\hline 0,04982 & 0,03180 & 0,04735 & 0,02933 & 0,04459 & 0,02657 & 0,04147 & 0,02345 & 0,03793 & 0,01991 \\
\hline 0,07416 & 0,04713 & 0,07179 & 0,04476 & 0,06911 & 0,04208 & 0,06606 & 0,03903 & 0,06256 & 0,03553 \\
\hline 0,10593 & 0,05188 & 0,10389 & 0,04984 & 0,10158 & 0,04753 & 0,09894 & 0,04489 & 0,09589 & 0,04184 \\
\hline 0,14595 & 0,07387 & 0,14448 & 0,07241 & 0,14281 & 0,07074 & 0,14091 & 0,06884 & 0,13871 & 0,06663 \\
\hline 0,19469 & 0,05055 & 0,19399 & 0,04984 & 0,19319 & 0,04905 & 0,19228 & 0,04814 & 0,19123 & 0,04709 \\
\hline 0,25218 & 0,00908 & 0,25236 & 0,00890 & 0,25256 & 0,00870 & 0,25280 & 0,00846 & 0,25307 & 0,00819 \\
\hline 0,31782 & 0,03854 & 0,31890 & 0,03962 & 0,32012 & 0,04084 & 0,32150 & 0,04222 & 0,32308 & 0,04380 \\
\hline 0,39034 & 0,06601 & 0,39222 & 0,06790 & 0,39433 & 0,07001 & 0,39672 & 0,07239 & 0,39943 & 0,07511 \\
\hline 0,46778 & 0,00832 & 0,47026 & 0,01080 & 0,47302 & 0,01356 & 0,47612 & 0,01666 & 0,47963 & 0,02017 \\
\hline 0,54758 & 0,14611 & 0,55036 & 0,14334 & 0,55344 & 0,14025 & 0,55688 & 0,13681 & 0,56075 & 0,13294 \\
\hline 0,62676 & 0,06694 & 0,62952 & 0,06418 & 0,63256 & 0,06113 & 0,63595 & 0,05775 & 0,63972 & 0,05397 \\
\hline 0,70223 & 0,09057 & 0,70467 & 0,08812 & 0,70735 & 0,08544 & 0,71032 & 0,08248 & 0,71361 & 0,07918 \\
\hline 0,77113 & 0,04869 & 0,77304 & 0,04678 & 0,77512 & 0,04470 & 0,77742 & 0,04240 & 0,77995 & 0,03987 \\
\hline 0,83120 & 0,00237 & 0,83247 & 0,00364 & 0,83385 & 0,00502 & 0,83536 & 0,00653 & 0,83702 & 0,00819 \\
\hline 0,88104 & 0,01085 & 0,88168 & 0,01021 & 0,88238 & 0,00952 & 0,88313 & 0,00876 & 0,88397 & 0,00793 \\
\hline 0,92025 & 0,00133 & 0,92037 & 0,00145 & 0,92051 & 0,00159 & 0,92065 & 0,00173 & 0,92081 & 0,00189 \\
\hline 0,94938 & 0,01459 & 0,94915 & 0,01481 & 0,94891 & 0,01505 & 0,94864 & 0,01532 & 0,94835 & 0,01561 \\
\hline 0,96972 & 0,01226 & 0,96933 & 0,01266 & 0,96889 & 0,01309 & 0,96842 & 0,01356 & 0,96789 & 0,01409 \\
\hline 0,98302 & 0,00104 & 0,98260 & 0,00062 & 0,98213 & 0,00015 & 0,98162 & 0,00037 & 0,98104 & 0,00094 \\
\hline 0,99112 & 0,00013 & 0,99076 & 0,00023 & 0,99036 & 0,00063 & 0,98990 & 0,00109 & 0,98939 & 0,00160 \\
\hline 0,99569 & 0,00470 & 0,99543 & 0,00444 & 0,99513 & 0,00414 & 0,99479 & 0,00380 & 0,99440 & 0,00340 \\
\hline 0,99807 & 0,00708 & 0,99791 & 0,00692 & 0,99771 & 0,00672 & 0,99748 & 0,00649 & 0,99721 & 0,00622 \\
\hline 0,99921 & 0,00822 & 0,99912 & 0,00812 & 0,99900 & 0,00801 & 0,99886 & 0,00787 & 0,99870 & 0,00771 \\
\hline 0,99971 & 0,00029 & 0,99966 & 0,00034 & 0,99960 & 0,00040 & 0,99952 & 0,00048 & 0,99943 & 0,00057 \\
\hline & 0,146114 & & 0,143337 & & 0,140254 & & 0,136811 & & 0,132941 \\
\hline & aderiu & & aderiu & 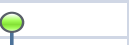 & aderiu & & aderiu & & aderiu \\
\hline & & beca & a fiou & & & & & & \\
\hline$(+)$ & & & & & & & & & \\
\hline
\end{tabular}

Figura 4. Planilha com as simulações seguintes, replicadas pela primeira simulação.

Figure 4. Spreadsheet with the next simulations, replicated by the first simulation.

\section{RESULTADOS E DISCUSSÃO}

\section{Definição do modelo final}

Pelas figuras 4 e 5 pode-se observar - pelas mensagens de 'aderiu' - que todas as 11 opções testadas em simulação logram êxito pelo teste de Kolmogorov-Smirnov. Ou seja, para todas as opções testadas para essa parcela de dados de campo, verifica-se que o valor de maior divergência não é significativo, o que indica que, nesse caso, há aderência dos dados à distribuição Weibull, com 99\% de probabilidade de acerto, para qualquer uma das opções.

Porém, a opção para $1,0 \mathrm{~d}_{\min }$, que recebe o símbolo (+), é a que deve ser escolhida, pois apresenta a menor das divergências, dentre todas as opções simuladas. A figura 6 ilustra o comportamento dessa função com os valores dos parâmetros $a, b$ e $c$ dessa melhor opção para a função Weibull. 


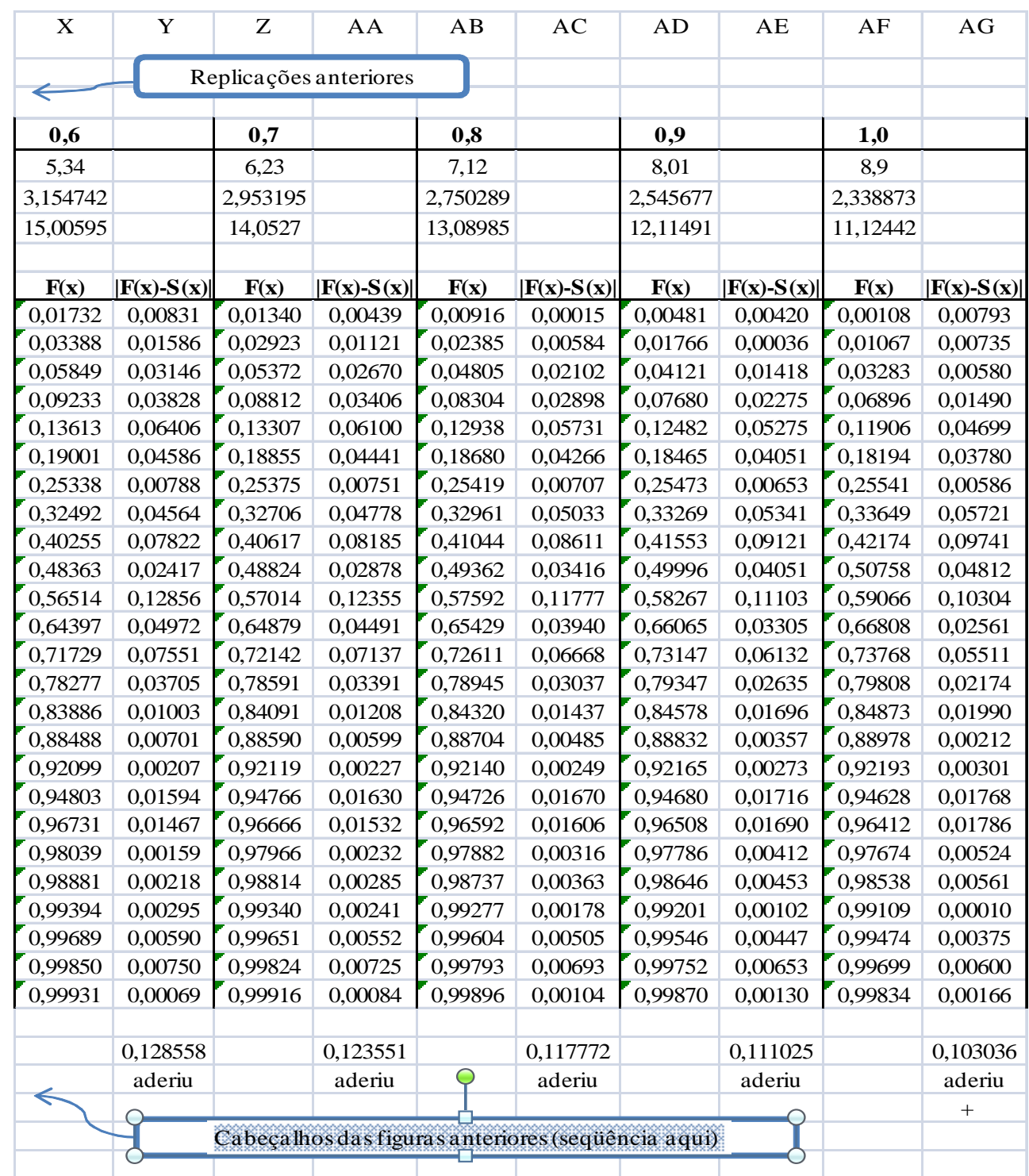

Figura 5. Planilha com simulações seguintes, replicadas pela primeira simulação (continuação da fig. 4). Figure 5. Spreadsheet with the next simulations, replicated by the first simulation (fig. 4 continuation).

\section{Avaliação do método}

Além da avaliação própria do método apresentado, com a simulação de suas alternativas de ajuste, uma medida suficiente para a sua ratificação é a de se fazer a sua avaliação geral, por comparação com outra forma de ajuste conhecida, particularmente com emprego de computadores, pois se propõe aqui uma metodologia baseada no uso desse recurso. Examinando-se os principais programas, ou pacotes estatísticos, para computador, pode-se perfeitamente aferir que nenhum deles executa o ajuste da função Weibull de maneira direta ou automática, seja pelo método dos percentis ou outro, da máxima verossimilhança ou dos momentos. Para esse propósito, há sempre que se classificar os dados da distribuição, calcular estimadores, organizar planilhas à parte para calcular teste de aderência por Kolmogorov-Smirnov etc. Alguns programas fornecem as curvas teóricas da função Weibull, mas nunca os parâmetros calculados. Enfim, esses pacotes podem ser usados, mas para se calcular os vários passos do método escolhido. 


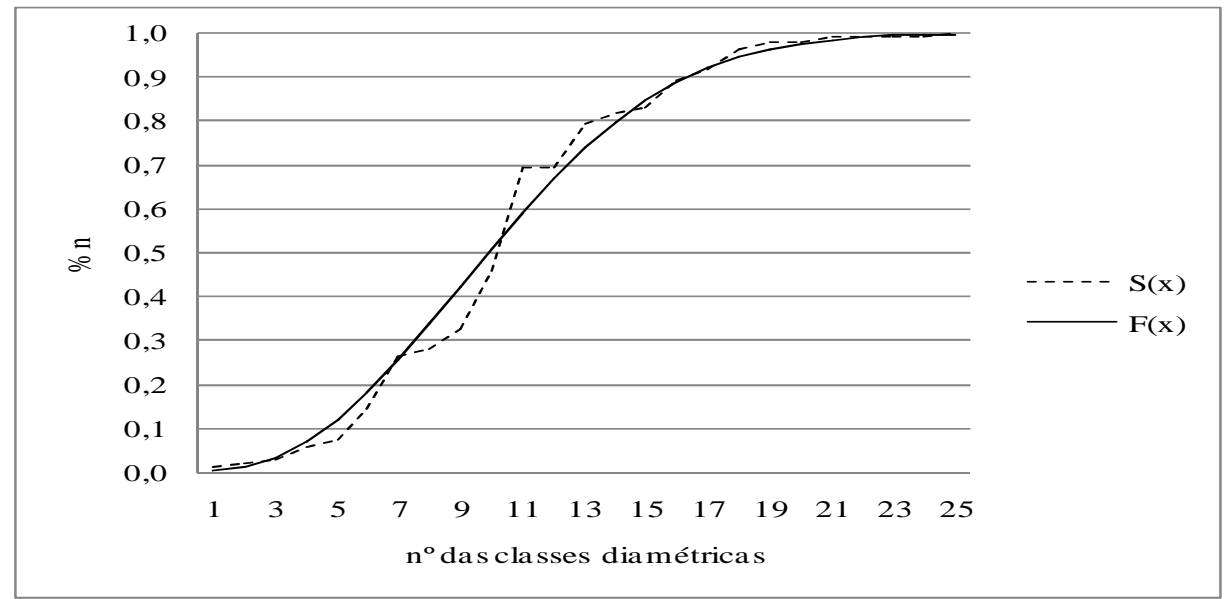

Figura 6. Comportamento da melhor opção para a função de distribuição de frequências Weibull, função Weibull escolhida $\mathrm{F}(\mathrm{x}) \mathrm{X}$ observada $\mathrm{S}(\mathrm{x})$.

Figure 6. Behavior of the best Weibull frequency distribution function option.

Para se fazer essa avaliação geral do método por comparação, e como aqui se propõe um método para uso por engenheiros florestais, lança-se mão então dos recursos comumente utilizados por eles para ajustar a função Weibull. Em pesquisa coloquial, nota-se que eles usam métodos de ajuste por regressão não linear, por pacotes de computador. Experimenta-se, então, ajustar os dados da mesma parcela aqui usada, por meio do programa Statistica, com o módulo para ajuste de modelos não lineares. Entretanto, por mais que se repitam os cálculos por diferentes atribuições de parâmetros iniciais, chamados de "sementes", recursos inerentes ao método de ajuste, os resultados são sempre muito ruins. Paradoxalmente, ao se optar por outro programa também bastante popular, chamado TableCurve $2 D$ v5, da AISN Software Inc., os resultados são então satisfatórios. E tal como no anterior, por mais que se variem as sementes, os valores de ajuste são sempre os mesmos, com mínimas e desprezíveis variações.

Para se explorarem os recursos do método de ajuste por regressão não linear, pede-se ao programa para calcular todos os três parâmetros - $a, c$ e $b-$, justamente para se testar se os resultados assemelham-se aos do método apresentado, no qual o parâmetro $a$ é lançado por simulação em 11 opções como porcentagens de $d_{\min }$ e os demais são calculados por fórmulas. Conclusivamente, os resultados obtidos são semelhantes, como se pode ver pela planilha apresentada na figura 7. Os valores dos seus três parâmetros são muito próximos aos obtidos na simulação do método dos percentis apresentado na opção de $1,0 \mathrm{~d}_{\min }$, escolhida lá como a melhor, tal como se pode comparar visualmente pela figura 5 . O programa usado mostra o coeficiente de variação $(\mathrm{CV} \%)$ - desvio padrão de estimativa em relação à média dos dados - de 5,7\%, valor baixo, que indica que o ajuste é bom.

Mas ao se examinar mais detalhadamente os dados da análise de Kolmogorov-Smirnov na figura 7 e compará-los aos da figura 5 do método aqui defendido, pode-se ver que o valor de maior divergência, apesar de indicar aderência da função aos dados pela confrontação ao valor tabelar, é $20 \%$ maior do que o obtido no método de simulação apresentado $(0,123579$ contra 0,103036$)$, o que indica uma mais bem ajustada função no método dos percentis. Essa vantagem pode ser visualizada ao se compararem os gráficos de comportamento das funçõe apresentados na figura 8 e na figura 6 . A diferença não deve ser creditada como pequena, pois, no caso do emprego da função em modelo de simulação florestal, essa precisão é fundamental. Vinte por cento podem significar praticamente o valor da aplicação de um desbaste florestal nessas classes diamétricas.

\section{Reaplicação do método}

Uma vez executado todo o algoritmo da metodologia apresentada e elaborada a planilha, pode-se facilmente fazer o ajuste da função Weibull para as demais parcelas do povoamento florestal em estudo. Para tal propósito, basta colocar cada parcela na planilha, em arranjo de substituição, e repetir os passos 1, 2, 3, 4, 5, 6 e 21 do algoritmo, com as devidas adaptações por diferenças em quantidade, tanto de árvores como de classes de DAP. Os demais passos são executados automaticamente, em adequação a cada nova parcela introduzida. 
Para todas as parcelas aplicadas com o método, a qualidade de precisão é inalterável. Todavia, para se avaliar mais uma vez o método por comparação, lança-se mão outra vez daquele pacote estatístico e ajustam-se os dados por regressão não linear, agora para a parcela 2 . Na tabela 3 , podem-se ver os dados desse ajuste e se nota que, mais uma vez, os resultados obtidos são semelhantes aos do método apresentado. Ou seja, os três parâmetros são muito próximos aos obtidos na melhor simulação do método dos percentis, agora na opção de $0,7 \mathrm{~d}_{\min }$. $\mathrm{O} \mathrm{CV} \%$ de 2,0, baixo para a média dos dados, também sinaliza um ajuste bom na regressão.

No entanto, no exame mais detalhado dos dados da análise de Kolmogorov-Smirnov e na comparação aos do método aqui defendido, pode-se ver que o valor de maior divergência, apesar de indicar também aderência da função aos dados pela confrontação ao valor tabelar, é agora $83 \%$ maior do que o obtido no método dos percentis apresentado, ou seja, 0,061846 contra 0,033872 . Então, no caso do emprego da função obtida por regressão em modelo de simulação florestal, essa diferença pode levar a um erro ainda maior no valor da aplicação de um desbaste florestal nessas classes diamétricas, do que o já mostrado com a primeira parcela.

\begin{tabular}{|c|c|c|c|c|c|c|c|c|c|}
\hline & \multicolumn{8}{|c|}{ Parâmetros da função Weibull ajustados por regressão } & \\
\hline & & & $\mathbf{d}_{\min }=$ & 8,9 & & & $a=$ & 8,9928 & \\
\hline & & & dmáx $=$ & 33 & & & $c=$ & 2,50023 & \\
\hline & & & & & & & $\boldsymbol{b}=$ & 10,47742 & \\
\hline \multicolumn{10}{|c|}{ C L A S S E S DAP (cm) } \\
\hline $\mathbf{n}^{\mathbf{o}}$ & lim.inf $\leq$ & centro & $<$ lim.sup & fo & acum & $\mathbf{S}(\mathbf{x})$ & & $\mathbf{F}(\mathbf{x})$ & $\mathbf{F}(\mathbf{x})-\mathbf{S}(\mathbf{x})$ \\
\hline $\mathbf{1}$ & 8,5 & 9 & 9,5 & 1 & 1 & 0,00901 & & 0,00052 & 0,00849 \\
\hline 2 & 9,5 & 10 & 10,5 & 1 & 2 & 0,01802 & & 0,00781 & 0,01020 \\
\hline 3 & 10,5 & 11 & 11,5 & 1 & 3 & 0,02703 & & 0,02761 & 0,00059 \\
\hline 4 & 11,5 & 12 & 12,5 & 3 & 6 & 0,05405 & & 0,06276 & 0,00870 \\
\hline 5 & 12,5 & 13 & 13,5 & 2 & 8 & 0,07207 & & 0,11428 & 0,04221 \\
\hline 6 & 13,5 & 14 & 14,5 & 8 & 16 & 0,14414 & & 0,18149 & 0,03735 \\
\hline 7 & 14,5 & 15 & 15,5 & 13 & 29 & 0,26126 & & 0,26210 & 0,00084 \\
\hline 8 & 15,5 & 16 & 16,5 & 2 & 31 & 0,27928 & & 0,35243 & 0,07316 \\
\hline 9 & 16,5 & 17 & 17,5 & 5 & 36 & 0,32432 & & 0,44790 & 0,12358 \\
\hline 10 & 17,5 & 18 & 18,5 & 15 & 51 & 0,45946 & & 0,54356 & 0,08410 \\
\hline 11 & 18,5 & 19 & 19,5 & 26 & 77 & 0,69369 & & 0,63473 & 0,05896 \\
\hline 12 & 19,5 & 20 & 20,5 & O & 77 & 0,69369 & & 0,71752 & 0,02383 \\
\hline 13 & 20,5 & 21 & 21,5 & 11 & 88 & 0,79279 & & 0,78923 & 0,00357 \\
\hline 14 & 21,5 & 22 & 22,5 & 3 & 91 & 0,81982 & & 0,84849 & 0,02867 \\
\hline 15 & 22,5 & 23 & 23,5 & 1 & 92 & 0,82883 & & 0,89524 & 0,06641 \\
\hline 16 & 23,5 & 24 & 24,5 & 7 & 99 & 0,89189 & & 0,93042 & 0,03853 \\
\hline 17 & 24,5 & 25 & 25,5 & 3 & 102 & 0,91892 & & 0,95566 & 0,03675 \\
\hline 18 & 25,5 & 26 & 26,5 & 5 & 107 & 0,96396 & & 0,97294 & 0,00897 \\
\hline 19 & 26,5 & 27 & 27,5 & 2 & 109 & 0,98198 & & 0,98419 & 0,00221 \\
\hline 20 & 27,5 & 28 & 28,5 & $\mathrm{O}$ & 109 & 0,98198 & & 0,99118 & 0,00920 \\
\hline 21 & 28,5 & 29 & 29,5 & 1 & 110 & 0,99099 & & 0,99530 & 0,00431 \\
\hline 22 & 29,5 & 30 & 30,5 & 0 & 110 & 0,99099 & & 0,99761 & 0,00662 \\
\hline 23 & 30,5 & 31 & 31,5 & 0 & 110 & 0,99099 & & 0,99885 & 0,00786 \\
\hline 24 & 31,5 & 32 & 32,5 & 0 & 110 & 0,99099 & & 0,99947 & 0,00848 \\
\hline \multirow[t]{6}{*}{25} & 32,5 & 33 & 33,5 & 1 & 111 & 1,00000 & & 0,99977 & 0,00023 \\
\hline & & & $\mathbf{n}=$ & 111 & & & & & \\
\hline & & & & & & \multicolumn{3}{|c|}{ Maior divergência $==>$} & 0,123579 \\
\hline & & & & & & \multicolumn{3}{|c|}{ Kolmogorov-Smirnov $=>$} & aderiu \\
\hline & & & & & & & & & \\
\hline & & & & & & \multicolumn{2}{|c|}{ Valor tabelar $=>$} & 0,154713 & \\
\hline
\end{tabular}

Figura 7. Planilha com a opção de ajuste de Weibull por método de regressão calculado por pacotes. Figure 7. Spreadsheet with the option for Weibull adjustment by calculated regression method packets. 


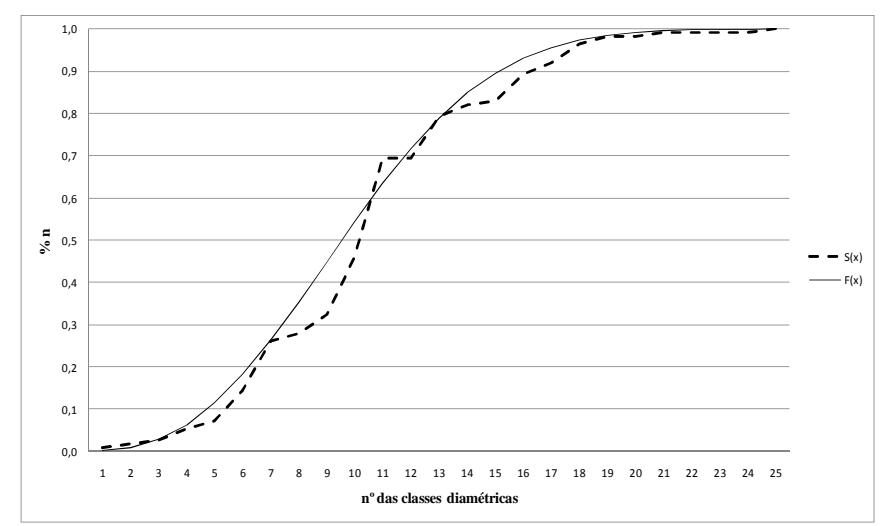

Figura 8. Comportamento da opção de Weibull ajustada por método de regressão calculado por pacotes, função Weibull ajustada $\mathrm{F}(\mathrm{x}) \mathrm{X}$ observada $\mathrm{S}(\mathrm{x})$.

Figure 8. Behavior of the option for Weibull adjustment by calculated regression method packets.

\section{Ratificação da qualidade de ajuste do método proposto}

Para consolidação final da metodologia, o método proposto e a validação, por comparação com o método coloquial de ajuste por regressão, são aplicados aleatoriamente em várias outras parcelas do povoamento florestal em diversas idades, como se pode ver na tabela 3 , em acréscimo às parcelas 1 e 2 já analisadas. Os resultados são sempre similares e quase sempre com vantagem ao método dos percentis. Essa persistência comportamental define um padrão de ajuste. Logicamente, pode-se observar que há algumas exceções, tais como nas parcelas 7 e 8 , fatos que se enquadram na aleatoriedade do processo.

Na coluna 'Vantagem' da tabela 3, vê-se a porcentagem de diferença entre os valores de maior divergência nas curvas da função, entre os dois métodos. Isso significa que na curva de $\mathrm{F}(\mathrm{x})$, que retrata os valores de frequências acumuladas, desde a primeira classe diamétrica até a classe de número mostrada na coluna 'Classe', verifica-se uma superestimação ou subestimação do número de árvores acumuladas nessas classes em relação ao método proposto. Os valores de porcentagem positivos mostram vantagem ao método dos percentis e vice-versa nos casos negativos. Observe-se que o fato de CV\% ser pequeno é sempre irrelevante.

Tabela 3. Demonstrativo geral da comparação dos métodos de ajuste testados, para várias parcelas.

Table 3. General statement of comparison of adjustment methods tested for several plots.

\begin{tabular}{|c|c|c|c|c|c|c|c|c|c|c|c|c|c|c|}
\hline \multicolumn{6}{|c|}{ Método proposto (percentis) } & \multirow{3}{*}{$\begin{array}{c}\text { Maior } \\
\text { di vergência }\end{array}$} & & \multicolumn{5}{|c|}{ Método alternativo (regressão) } & & \multirow[b]{3}{*}{$\mathrm{CV} \%$} \\
\hline \multicolumn{2}{|c|}{ Parcelaidade } & \multicolumn{3}{|c|}{ Parâmetros } & \multirow[b]{2}{*}{$\boldsymbol{b}$} & & \multirow[b]{2}{*}{ Classe } & \multirow{2}{*}{\begin{tabular}{|c|} 
Vantagem \\
$\%$ \\
\end{tabular}} & \multicolumn{2}{|c|}{ Parâmetros } & \multirow[b]{2}{*}{$\boldsymbol{b}$} & \multirow{2}{*}{\multicolumn{2}{|c|}{\begin{tabular}{|c|} 
Maior \\
divergência Classe
\end{tabular}}} & \\
\hline$n^{\circ}$ & (anos) & $\% \mathbf{d}_{\mathbf{m}}$ & $a$ & $c$ & & & & & $a$ & $c$ & & & & \\
\hline 1 & 13,1 & 1,0 & 8,9 & 2,3389 & 11,1244 & 0,103036 & 11 & 19,9 & 8,9928 & 2,5002 & 10,4774 & 0,123579 & 9 & 5,7 \\
\hline 2 & 13,1 & 0,7 & 6,02 & 2,7859 & 12,3752 & 0,033872 & 7 & 82,6 & 5,594 & 3,0031 & 12,3001 & 0,061846 & 7 & 2,0 \\
\hline 3 & 6,2 & 1,0 & 2,9 & 4,5596 & 10,4903 & 0,064074 & 7 & 62,4 & 2,9743 & 5,0879 & 9,8121 & 0,104047 & 9 & 5,9 \\
\hline 4 & 5,4 & 0,0 & 0,0 & 7,0101 & 13,8294 & 0,062917 & 7 & 66,4 & 1,467 & 6,1319 & 12,0153 & 0,104723 & 7 & 4,8 \\
\hline 5 & 6,2 & 0,5 & 3,5 & 4,6458 & 12,1524 & 0,060648 & 5 & 67,8 & 6,278 & 3,2396 & 9,0553 & 0,101764 & 9 & 4,8 \\
\hline 6 & 5,4 & 0,0 & 0,0 & 7,7038 & 16,5585 & 0,044993 & 3 & 88,5 & $-28,75$ & 20,388 & 44,987 & 0,084821 & 8 & 4,5 \\
\hline 7 & 19,0 & 0,0 & 0,0 & 5,3372 & 23,0619 & 0,080731 & 11 & $-7,1$ & $-18,44$ & 10,628 & 41,673 & 0,075033 & 11 & 3,4 \\
\hline 8 & 18,0 & 0,4 & 5,24 & 3,7342 & 18,7468 & 0,060464 & 4 & $-5,5$ & 10,55 & 2,4719 & 13,385 & 0,057151 & 12 & 3,9 \\
\hline 9 & 19,0 & 0,7 & 9,17 & 3,2157 & 15,2935 & 0,037430 & 10 & 83,0 & 11,03 & 2,5282 & 12,819 & 0,068503 & 13 & 2,5 \\
\hline 10 & 21,0 & 0,6 & 8,04 & 3,335 & 15,8561 & 0,043581 & 9 & 53,8 & 12,34 & 2,1701 & 11,057 & 0,067021 & 12 & 2,9 \\
\hline
\end{tabular}

\section{CONCLUSÕES}

- As formas corretas de calcular os parâmetros da função Weibull são os métodos da máxima verossimilhança, dos momentos e dos percentis, equivalentes na eficiência. A opção, neste trabalho, pelo método dos percentis fundamenta-se no propósito favorável do uso dos seus estimadores em cenários de prognose ou projeção da produção florestal, tal como indica Wendling (2007). Por isso, a comparação do método dos percentis com outra forma coloquial de ajuste é oportuna. O método 
alternativo de ajuste por regressão não linear, por pacotes computacionais, mostra-se pelos resultados que mostram que, apesar do seu bom desempenho, as funções assim obtidas não descrevem a estrutura da distribuição diamétrica tão bem quanto no método "clássico".

- Na função ajustada por regressão, o fato do CV\% sempre apresentar valores satisfatórios indica que o modelo de função ajusta bem, porém o resultado da distribuição dos resíduos também reflete as compensações - em algumas classes a função pode ajustar mal para cima e, em outras, mal para baixo da curva -, o que resulta em valores baixos para o total. Todavia, no emprego da função para simulação da produção florestal, ao se planejarem desbastes seletivos, no qual queira se cortar, por exemplo, parte da classe social 'dominada' e esta estiver subestimada ou superestimada pela função, então essa diferença pode induzir a erro no peso de desbaste.

- A simulação por arranjos de valores fixados para o parâmetro $a$, vinculando-o a um percentual do diâmetro mínimo do povoamento, $\mathrm{d}_{\min }$, em 11 opções de alternativas para a seleção da melhor função, é o fator de sucesso do método. Os parâmetros $a, c$ e $b$ têm cada qual o seu papel na definição da curva. Por isso devem ser tratados individualmente e não em conjunto, como no método da regressão. O parâmetro $a$ define a locação no início da curva, por isso é apropriado vinculá-lo ao $\mathrm{d}_{\min }$. Os demais parâmetros são calculados após a definição de cada opção do parâmetro $a$, e a melhor função é escolhida dentre essas opções.

- Após o cálculo da melhor opção de função Weibull por parcela, recomenda-se aprofundar a pesquisa, na escolha da melhor opção do seu parâmetro $a$, na situação por classe de sítio, processo adotado em Wendling (2007). É de se esperar, pela lógica do manejo florestal, que os parâmetros da função Weibull reflitam situações divergentes em diferentes classes de sítio. Para esse propósito, o modelo é adequado, devido à sua flexibilidade para assumir diferentes formas e assimetrias, ante ao seu emprego em variadas apresentações de dados.

- A planilha gerada pelo algoritmo apresentado serve para organizar, sistematizar e automatizar o processamento dos dados. Uma vez feita a montagem da planilha inicial, o seu emprego para as demais parcelas é tarefa menos árdua e possibilita fácil manuseio para o trabalho corriqueiro do engenheiro florestal.

\section{REFERÊNCIAS}

BAILEY, R. L. Weibull model for Pinus radiata diameter distribution. In: IUFRO Conference of the Advisory Group of Forest Statisticians, 1973. Statistics in Forest Research. Vancouver, 1973. p. 51 - 59.

BARRA, O. S. V.; SANQUETTA, C. R.; ARCE, J. E.; MACHADO S. do A.; CÔRTE, A. P. D. Proposta metodológica para o ajuste ótimo da distribuição diamétrica Weibull 3 p. Floresta, Curitiba, v. 34, n. 3, 2004.

GLADE, J. E. Prognose de volume por classes diamétricas para Eucalyptus grandis Hill Ex-Maiden. 128 f. Dissertação (Mestrado em Ciências Florestais) - Setor de Ciências Agrárias, Universidade Federal do Paraná, Curitiba, 1986.

GUIDORIZZI, H. L. Um curso de cálculo. 5. ed. São Paulo: LTC, 2001. v. 1 - 4.

HALDAR, A.; MAHADEVAN, S. Probability, reliability and statistical methods in engineering design. New York: J. Wiley \& Sons, 2000.

MALTAMO, M.; KANGAS, A.; UUTTERA, J. Comparison of percentile based prediction methods and the Weibull distribution in describing the diameter distribution of heterogeneous Scots pine stands. Forest Ecology and Management, Amsterdam, v. 133, n. 3, p. 263 - 274, 2000.

MICROSOFT CORPORATION. Microsoft Office Excel 2007. Microsoft Customer Service and Support, One Microsoft Way, Redmond, WA 98052-6399; www.microsoft.com/info/nareturns.htm. Manual de ajuda off-line do Excel.

SCOLFORO, J. R. S. Modelagem do crescimento e da produção de florestas plantadas e nativas. Lavras: UFLA/FAEPE, 1998. 
SOARES, T. S.; LEITE, H. G.; VALE, A. B.; SOARES, C. P. B.; SILVA, G. F. Avaliação de um modelo de passo invariante na predição da estrutura de um povoamento de Eucalyptus sp. Revista Árvore, Viçosa, v. 31, n. 2, 2007.

WENDLING, W. T. Sistema computacional e modelagem para simulação dinâmica da produção florestal. 191 p. Tese (Doutorado em Ciências Florestais) - Setor de Ciências Agrárias, Universidade Federal do Paraná, Curitiba, 2007. 